\title{
LA FORMALIZZAZIONE MATEMATICA NELLE TEORIE ECONOMICHE: PROBLEMI E PROSPETTIVE
}

\author{
GIOVANNI MARSEGUERRA (*)
}

Because the mathematical method is so powerful and beautiful and its possession still sufficiently rare to command distinction,

the mathematical economist is under constant temptation

to use it just for the sake of using it. [George Stigler, 1949]

RIASSUNTO. - All'interno della comunità degli economisti sembra essere oggi presente un crescente disagio e un diffuso malcontento relativamente alle capacità della modellistica neoclassica di comprendere e spiegare i fenomeni economici. La relazione intende esaminare criticamente il contributo della formalizzazione matematica nelle teorie economiche, mettendo in evidenza sia punti di forza di un tale approccio sia le debolezze insite in un'analisi che rischia spesso di privilegiare la bellezza dello strumento rispetto all'oggetto di studio. Si considerano poi alcune delle moderne prospettive per l'indagine economica offerte dalla modellistica basata sulla simulazione a computer che consente di tener conto della complessità della realtà in misura maggiore di un approccio puramente analitico. Viene infine evidenziata l'importanza di garantire la coesistenza di una pluralità di scuole di pensiero in economia e si esaminano altresì le difficoltà per la valutazione della ricerca provocate dall'esistenza di un paradigma dominante. Nelle conclusioni viene enfatizzata la necessità di considerare l'economia come una vera scienza sociale.

$* * *$

ABSTRACT. - There is today a widespread discontent within the international economists' community as far as the capacity of the dominant neoclassical paradigm both to

(*) Dipartimento di Discipline Matematiche, Finanza Matematica ed Econometria, Centro di Ricerche in Analisi Economica (CRANEC), Università Cattolica del Sacro Cuore. 
enhance our knowledge of economic phenomena and increase our capacity of governing the real economy. Somehow surprisingly, the lack of explicative relevance of the theory went almost hand in hand with the massive use of mathematics to formalize the theory. In order to investigate this peculiar feature of the evolution of economic science, this paper examines the contribution of mathematical formalization to the development of economic theory in the last sixty years pointing out both weaknesses as well as merits of a quantitative approach to economics. Finally, the need to consider economics as a truly social science si strongly emphasized.

\section{PREMESSA}

In questo saggio ci proponiamo di esaminare la relazione tra scienza economica e formalizzazione matematica nella convinzione che si tratti di un rapporto che si è dimostrato assai fecondo nel passato ma che oggi, pur mantenendo una rilevanza assolutamente primaria nella elaborazione della teoria economica, necessiti di un approfondito ripensamento alla luce del crescente disagio e della diffusa insoddisfazione oggi presenti nella comunità degli economisti in riferimento alla capacità della teoria di comprendere e spiegare la realtà economica. In effetti, tra le tante contraddizioni che hanno caratterizzato l'evoluzione della scienza economica degli ultimi sessanta anni, quella che più colpisce per la sua consistenza è costituita proprio dalla progressiva perdita di capacità della teoria di interpretare comprensibilmente la realtà, come se i cultori della dismail science avessero perso la consapevolezza che la scienza economica è una scienza sociale e il fine ultimo delle teorie economiche è quello di comprendere il passato, decifrare e governare il presente e possibilmente prevedere il futuro. $\grave{E}$ interessante osservare come, almeno in termini puramente temporali, questa perdita di rilevanza esplicativa delle teorie economiche si sia realizzata contemporaneamente al periodo di massimo utilizzo dello strumento matematico. Non è quindi inutile chiedersi, come faremo in questo saggio, se questa contemporaneità sia stata o meno casuale (senza pretendere, peraltro, di trovare risposte definitive).

Il presente lavoro, che si propone di esaminare il ruolo della formalizzazione matematica nella teoria economica sia in una prospettiva storica sia in riferimento alle potenzialità oggi offerte alla scienza economica dall'utilizzo della straordinaria capacità di calcolo e di simulazione dei moderni computer elettronici, si compone di cinque parti: dopo la Premessa, nella Sezione 2 verranno esaminate le attuali diffi- 
coltà della teoria economica nella capacità di comprendere la realtà e, anche attraverso citazioni di alcuni eminenti economisti, il crescente distacco tra modellistica teorica e concreti problemi economico-sociali; la Sezione 3 esamina il contributo della matematica alla evoluzione della scienza economica negli ultimi sessanta anni; la Sezione 4 presenta, in termini necessariamente molto sintetici, un recente e innovativo approccio alla modellistica economica basato sulle simulazioni numeriche. La Sezione 5 infine presenta alcune riflessioni conclusive

\section{MOdELlistica TEORICA E CONOSCENZA ECONOMICA}

In termini molto generali l'obiettivo della scienza economica consiste (o perlomeno dovrebbe consistere) nella conoscenza della realtà economica. Il fine ultimo di questa conoscenza poi è (o dovrebbe) essere rappresentato dal miglioramento della capacità dell'uomo di controllare questa realtà. Dunque conoscenza positiva ma anche normativa, ovvero l'economia politica e la politica economica. A partire da queste premesse, il metodo matematico si presenta naturalmente come lo strumento ideale per far avanzare la conoscenza, in analogia con quanto successo con altre discipline come la fisica, la chimica, la biologia e via discorrendo. Ed in effetti, come si argomenterà più in dettaglio nella successiva Sezione 3, questo è esattamente lo spirito con il quale è stata utilizzata la matematica da intere generazioni di economisti, a partire dai pionieri del diciannovesimo secolo. Tuttavia negli ultimi decenni, l'uso della matematica è diventato così pervasivo della teoria economica non solo da offuscare sotto molto profili la ragione ultima che dovrebbe governare l'adozione di un tale strumento, vale a dire la conoscenza della realtà, ma addirittura frequentemente la matematica ha smesso di essere strumento per diventare essa stessa obiettivo della ricerca economica. Evidentemente, con un utilizzo così distorto del metodo matematico, la capacità della teoria di spiegare la realtà risulta gravemente compromessa. Come ammonisce correttamente Paolo Sylos Labini:

"Per uno sviluppo scientifico ci vogliono due "erre", non una, cioè rigore, che può essere dato appunto dai metodi matematici, e rilevanza, cioè i problemi devono essere reali. Il rigore senza la rilevanza porta solo a modelli sterili, magari eleganti dal punto di vista formale, ma inutili per la comprensione della realtà". (Sylos Labini, 2002). 
Una certa insofferenza per l'incapacità della teoria di ampliare la conoscenza della realtà è in verità presente da sempre nella comunità degli economisti (potremmo dire che è connaturata alla professione), e tuttavia negli ultimi decenni questa scontentezza si è fatta più acuta e diffusa. Valga tra tutte la testimonianza sconsolata di Mark Blaug, uno dei più seri e attenti economisti di origine olandese:

"..modern economics is sick; economics has increasingly become an intellectual game played for its own sake and not for its practical consequences". (Blaug, 1997).

Anche Edmond Malinvaud, uno dei maggiori economisti francesi del Novecento, esprime la stessa preoccupazione:

"The risk [..] seriously exists that the discipline progressively loses touch with real problems, develops on its own into a scholastic and becomes less and less significant for the layman's concerns". (Malinvaud, 1991).

Le riflessioni sopra riportate possono indurre nella tentazione di ritenere che le preoccupazioni sull'evoluzione della teoria economica appartengano solamente ad economisti di origine non anglosassone. ${ }^{1}$ In realtà non è difficile trovare analoghe preoccupazioni anche in affermati economisti inglesi come John Hey e americani come Ariel Rubinstein. Hey ad esempio, nel tracciare un bilancio della sua attivatà come Managing Editor dell'Economic Journal, così scrive:

"Many of the submissions [to The Economic Journal] do not appear to be written in order to further economic knowledge. [..] it is [..] disheartening that so many economist seem to be playing "the journal game", i.e. producing variations on a theme that are uninteresting and which do not enlighten." (Hey, 1997).

E Rubinstein, uno dei più influenti economisti degli ultimi cinquant'anni nell'ambito della teoria dei giochi, è straordinariamente efficace e poetico ad un tempo:

"The word model sounds more scientific than fable or fairy tale although I do not see much difference between them". (Rubinstein, 2006).

1 Sebbene Blaug, nato a L'Aia, sia poi diventato cittadino britannico. 
Come si accennava nella Premessa, questa perdita di capacità esplicativa della teoria economica è andata pari passo con l'utilizzo sistematico del metodo matematico che, da strumento che era inizialmente, si è via via trasformato impropriamente quasi nel fine ultimo della ricerca economica. Testimonianza efficace di questo avvitamento della teoria su se stessa è il passo seguente di Micho Morishima, uno dei più stimati ed affermati economisti del secondo dopoguerra:

"...the reason for present-day economics having lapsed into the wretched state of affairs we have noted above is the fact that so deep and extensive bas been the mathematization of economics since 1940 that it has lost all sense of balance, becoming divorced from knowledge of economic systems and economic history. There is only one medicine which will cure this malaise, and that is for the theorists to make a serious effort in the direction of the institutionalization of economics, in the sense of slowing the speed of all development towards mathematization and developing economic theory in accordance with knowledge of economic organizations, industrial structure and economic histo$r y$ [Morishima, 1984, p. 70].

Pochi anni dopo queste desolate riflessioni di Morishima, Maurice Allais, vincitore del Premio Nobel per l'Economia nel 1988, è forse ancora più incisivo:

"For almost fifty years contemporary economic literature had developed too often in a totally erroneous direction with the construction of completely artificial mathematical models detached from reality; and too often it is dominated more and more by a mathematical formalism which fundamentally represents an immense regression" [Allais, 1989, p. 13].

Alla luce delle riflessioni sopra riportate sembra dunque importante esaminare, anche in una prospettiva storica, le modalità attraverso le quali il formalismo matematico si è ridotto, secondo Allais, ad una "gigantesca regressione".

\section{LA MATEMATICA IN ECONOMIA}

Anche se già molti economisti del XVIII secolo si erano avvalsi della formalizzazione matematica per lo studio dei fenomeni economici, tuttavia si tende formalmente a far coincidere l'inizio delle applicazioni della matematica all'economia con la pubblicazione del lavoro 
Recherches sur les principes mathematiques de la teorie de richesses da parte di Augustin Cournot nel 1838, mentre i fondatori dell'economia matematica sono unanimemente riconosciuti in Walras (1874-77), Edgeworth (1881), Marshall (1890) e Pareto (1909). In realtà tutti i grandi economisti della seconda metà dell'Ottocento mostrano grande competenza matematica.

Date le grandi potenzialità della matematica, e in particolare del simbolismo matematico come linguaggio della scienza, non desta sorpresa la sua utilizzazione per comprendere i fenomeni economici. I vantaggi derivanti dall'utilizzo della matematica sono in effetti molteplici. Se si vuole modellare la realtà con i simboli della matematica, si è obbligati a definire e specificare con grande precisione ogni grandezza che si vuole rappresentare, conseguendo in tal modo la massima chiarezza di concetto. ${ }^{2}$ Con le parole del grande matematico Fourier:

"Il suo [della matematica] attributo principale è la chiarezza: essa non ha simboli per le idee confuse". (Jean Baptiste Joseph (Baron) Fourier, Théorie analitique de la chaleur, 1822).

Grazie alle leggi interne della matematica poi si può raggiungere la conclusione di un ragionamento con assoluta certezza e massimo rigore metodologico. Inoltre ogniqualvolta si sia riusciti a risolvere un problema in riferimento ad un determinato fenomeno, ogni altro fenomeno formalizzabile con gli stessi simboli può anch'esso essere considerato risolto. Dunque chiarezza, rigore e generalità. Tutto ciò è riassunto in modo straordinariamente incisivo in un passo contenuto in uno dei primi e più autorevoli manuali di Economia matematica pubblicati in Italia:

".... l'uso delle leggi interne di un qualsiasi simbolismo matematico richiede, se fatto correttamente, che sia precisata esplicitamente ogni ipotesi che viene considerata come fondamentale per lo svolgimento del ragionamento. In altre parole l'uso del linguaggio matematico nella rappresentazione degli enti di una scienza qualsiasi viene ad accostare tale scienza all'ideale della esposizione matematica, ideale che è rappresentato dal sistema assiomatico. Intendiamo con questa espressione indicare non un sistema logico nel quale le proposizioni ini-

2 Questa chiarezza dovrebbe poi essere, in linea di principio, a tutto vantaggio della comprensione della realtà. Come vedremo, tuttavia, non sempre è questo il caso. 
ziali sono imposte autocraticamente dal di fuori, ma un sistema nel quale tutte le ipotesi vengono esplicitamente enunciate all'inizio della trattazione, tutti $i$ termini usati sono enunciati come primitivi oppure esplicitamente definiti, tutte le regole di deduzione sono esplicitamente formulate". (C.F. Manara e P.C. Nicola, 1967).

Vengono qui sinteticamente ed efficacemente descritte le caratteristiche principali di una impostazione deduttivo-assiomatica, perché a partire da un numero limitato di assiomi (ipotesi) l'analisi si sviluppa attraverso una catena di deduzioni, ed astratta, perché non sono richiesti riferimenti istituzionali precisi, dai quali anzi si prescinde completamente. ${ }^{3}$ Questo tipo di impostazione, che è tipico della moderna teoria economica nella versione neoclassica, consente (e di fatto negli ultimi cinquant'anni ha consentito) di costruire sviluppi analitici assai sofisticati. Tuttavia, per costruire questi raffinati modelli, si devono necessariamente introdurre, presto o tardi, cosiddette "ipotesi ad boc", indispensabili per semplificare la trattazione. Queste ipotesi, rispondendo a richieste di trattabilità matematica del modello, molto spesso hanno una giustificazione empirica assai limitata o addirittura discutibile. $\mathrm{Ma}$ partendo da ipotesi discutibili anche le conclusioni, peraltro assolutamente indiscutibili in virtù della coerenza interna delle leggi matematiche, perdono di rilevanza empirica. Dunque, quando le esigenze del formalismo matematico prevalgono su quelle del ragionamento economico, il modello rischia di perdere ogni capacità esplicativa della realtà. Il che rimanda in modo immediato alle citazioni riportate nella Sezione precedente.

Le difficoltà sopra descritte sono colte in modo molto preciso da Gerard Debreu, matematico e premio Nobel per l'Economia, uno delle menti più brillanti nel campo della ricerca economica degli ultimi sessant'anni, che nella voce Mathematical economics del Palgrave pubblicata nel 1987 così scrive:

"Quando un modello formale di una economia assume vita propria, esso diventa l'oggetto di un processo inesorabile, nel quale vengono perseguiti senza sosta il rigore, la generalità e la semplicità. [...] L'interpretazione economica dei teoremi cosi ottenuti è l'ultimo passo dell'analisi. [...] .. una teoria assiomatiz-

3 Per una presentazione rigorosa e sistematica della moderna economia matematica si può fare riferimento a P.C. Nicola, 1983, 2000. 
zata ba una forma matematica completamente separata dal suo contenuto economico. [...] Se si rimuove l'interpretazione economica dei concetti primitivi, delle ipotesi e delle conclusioni del modello, la sua nuda struttura matematica deve comunque reggere". (G. Debreu, 1987).

$\grave{E}$ in sostanza un elogio della bellezza irresistibile della forma, con il contenuto economico che diventa essenzialmente irrilevante. L'attrazione della forma può poi arrivare ad un tal punto che se per un problema economico non si trova una adeguata rappresentazione matematica, questo problema viene accantonato a vantaggio di quelli riducibili alla forma matematica. Non solo quindi al grande rigore formale del modello può corrispondere una assoluta irrilevanza empirica dei risultati (in forza di ipotesi irrealistiche), ma addirittura può essere il modello stesso ad affrontare una questione di scarsa rilevanza (ma formalizzabile in modo impeccabile), riproponendo così l'annoso problema del conflitto tra matematica e realtà, come icasticamente segnalava già Einstein:

"As far as the laws of mathematics refer to reality, they are not certain; and as far as they are certain, they do not refer to reality". (A. Einstein, citato in J. R. Newman, The World of Mathematics, New York, 1956).

Come uscire da questo impasse tra matematica e realtà, tra teoria e dato empirico? Si tratta evidentemente di ripartire dalle conoscenze che sono necessarie per fare della buona teoria economica, ovvero dalle caratteristiche che fanno un buon economista. Si tratta di un insieme composito di competenze, tra le quali è opportuno segnalare:

i) La conoscenza dei fattori storici, sia di natura economica che extraeconomica. Come già ricordava Schumpeter:

"la massima parte degli errori fondamentali comunemente commessi nell' analisi economica è dovuta alla mancanza di esperienza storica più che a qualsiasi altra deficienza nel corredo scientifico dell'economista". (Schumpeter 1959, p. 16);

ii) La conoscenza delle istituzioni: architettura, modalità di funzionamento, interazione tra diversi organismi e tra questi e la società, dunque la conoscenza della storia delle istituzioni;

iii) La conoscenza del linguaggio della matematica e l'applicazione del suo rigore: come testimonia uno dei massimi economisti italiani, Luigi Pasinetti, in riferimento specificatamente alla famosa contro- 
versia sul capitale tra le due Cambridge (quella in Inghilterra e quella negli USA):

".. dobbiamo riconoscere che sarebbe stata impensabile una critica tanto serrata alla teoria economica dominante - soprattutto sarebbe stata impensabile l'accettazione dei risultati di questa critica - senza l'utilizzo della notazione matematica, per il tramite della quale $i$ contendenti si sono potuti confrontare con un linguaggio comune" (L.L. Pasinetti, 2003);

iv) La conoscenza del diritto e di come gli aspetti legali e regolamentali influiscono sui processi economici;

v) La conoscenza della società;

vi) La conoscenza della psicologia.

L'elenco è però ovviamente incompleto perché quello che serve è, in via di sintesi, un approccio davvero interdisciplinare, l'esatto contrario di quanto molto spesso avviene oggi, anche nelle più rinomate scuole americane di Ph.D.

Le riflessioni sopra riportate sono mirabilmente sintetizzate da Keynes che, nell'epitaffio scritto per Alfred Marshall, e pubblicato per la prima volta nel 1924, così scrive:

"... the master-economist must possess a rare combination of gifts. He must reach a bigh standard in several different directions and must combine talents not often found together. He must be mathematician, historian, statesman, philosopher - in some degree. He must understand symbols and speak in words. He must contemplate the particular in terms of the general, and touch abstract and concrete in the same flight of thought. No part of man's nature or his institutions must lie entirely outside his regard. He must be purposeful and disinterested in a simultaneous mood, as aloof and incorruptible as an artist, yet sometimes as near the earth as a politician' (Keynes, 1933, p. 141).

Anche Luigi Einaudi, in un saggio scritto nel 1936, coglie con grande efficacia la natura davvero multidisciplinare del mestiere dell'economista:

"...La manovra monetaria opera su un congegno delicatissimo e complicatissimo: e riesce a quel manovratore il quale alla chiarezza delle idee astratte sa unire, rapidissimo, l'intuito dei fatti invisibili". (L. Einaudi, 1936). 


\section{I MODELLI BASATI SULLA SIMULAZIONE ${ }^{4}$}

Si può dire che ogni scienza che voglia cercare di comprendere il suo oggetto di indagine deve inevitabilmente ricorrere all'uso di modelli, vale a dire a rappresentazioni semplificate di quello che intende studiare. La scienza economica, almeno in questo senso, non fa eccezione e utilizza regolarmente modelli volti a fornire rappresentazioni semplificate della realtà. Sebbene in economia la maggior parte di questi modelli siano costruiti mediante termini matematici, esistono tuttavia diverse classi (o tipologie) di modelli che possono essere utilizzati da una scienza. In termini generali, seguendo Terna (2003 e 2005) e facendo riferimento all'impostazione ormai classica di Oström (1988), possiamo identificare tre tipologie di modelli (o sistemi simbolici):

i) i modelli letterario-descrittivi;

ii) i modelli matematico-statistici;

iii) i modelli basati sull'utilizzo della simulazione al computer.

I primi sono caratterizzati dal massimo della flessibilità, nel senso che tramite la descrizione verbale è possibile conseguire un elevato livello di dettaglio e precisione nella descrizione dei fenomeni economici studiati. Tuttavia, mancando di quei meccanismi di controllo interno e di verifica garantiti dall'utilizzo della matematica, nell'uso di questa tipologia modellistica è alto il rischio di incoerenze e salti logici. I secondi sono i modelli esaminati nelle precedenti Sezioni, cioè quelli tradizionali dell'impostazione neoclassica dell'ultimo mezzo secolo, che si portano dietro però il problema delle semplificazioni e quindi il rischio dell'allontanamento da quella realtà che vorrebbero descrivere.

I modelli basati sull'impiego del computer possono essere effettivamente considerati come la versione moderna dell'utilizzo della matematica per comprendere la realtà. Tuttavia, per una corretta descrizio-

4 Per un'analisi approfondita della modellistica teorica succintamente richiamata in questa Sezione, così come anche per una sua valutazione critica, si può fare riferimento ad una serie di contributi di Pietro Terna che, di questo approccio, è uno dei massimi esperti a livello internazionale. Si vedano in particolare Terna 2003, 2005: Terna, Boero, Morini e Sonnessa, 2006. A questi lavori anche noi ci siamo ispirati per l'esposizione che segue. 
ne di quest'ultima tipologia modellistica, è necessario premettere che, in prima approssimazione, vi sono (almeno) due diverse e spesso complementari modalità di impiego del computer per investigare la realtà economica: la prima consiste nell'utilizzo delle potenzialità di calcolo del computer per ottenere le soluzioni di un modello analitico non risolvibile formalmente (essendo quest'ultimo il mezzo attraverso il quale si cerca di conoscere il fenomeno economico); nella seconda modalità invece è la simulazione stessa che viene utilizzata come strumento di indagine della realtà. La prima modalità non è altro dunque che la naturale evoluzione dei modelli matematici standard, nel senso che lo sviluppo di modelli sempre più raffinati e matematicamente sofisticati ha di fatto richiesto l'utilizzo di capacità di calcolo sempre più elevate, conseguibili unicamente grazie alle straordinarie potenzialità dei moderni computer. Si fa qui più precisamente riferimento all'utilizzo del computer per il trattamento con metodi numerici dei sistemi non lineari, delle equazioni alle differenze, delle equazioni differenziali (incluse le equazioni alle derivate parziali e le equazioni differenziali stocastiche), e via discorrendo. Rientrano in questa tipologia di utilizzo del computer anche la determinazione delle posizioni di equilibrio nei modelli tradizionali, la risoluzione di problemi di ottimizzazione mediante la programmazione dinamica, i metodi di perturbazione, l'analisi di sensitività e anche la simulazione con la metodologia Monte Carlo della dinamica temporale di sistemi di equazioni stocastiche. Come già indicato questo utilizzo del computer, secondo l'impostazione neoclassica, funge da utile complemento ai modelli analitici (invariabilmente ritenuti più rigorosi).

La seconda modalità di utilizzo del computer, quella realmente innovativa e che ricorre all'uso della simulazione non come complemento di un precedente apparato analitico (il vero modello esplicativo!) ma considerando la stessa simulazione come strumento di indagine della realtà, è quella che abbiamo sopra identificato come terzo sistema simbolico. I modelli della cosiddetta Agent-based Computational Economics (ACE) sono costruiti a partire dalla definizione delle regole che stabiliscono il comportamento dei singoli agenti in un ambiente complesso e sono costituiti da agenti che agiscono e interagiscono tra loro e con l'ambiente. I modelli che utilizzano la simulazione via computer secondo questa seconda impostazione possono dunque essere considerati una ulteriore importante evoluzione nella costruzione di modelli della realtà sociale. Seguendo Gottardi e Babetto, 
"la Agent-based Computational Economics (ACE) è venuta negli ultimi anni a proporsi come branca, o vera e propria disciplina secondo i suoi cultori, dedicata alla modellizzazione al computer di economie intese come sistemi evolutivi generati dall'interazione di agenti indipendenti. L'obiettivo fondamentale di ACE è comprendere la formazione apparentemente spontanea di regolarità nei processi economici, come il coordinamento non pianificato delle attività, che nelle economie di mercato gli economisti associano alla mano invisibile di Adam Smith. La sfida è spiegare come queste regolarità emergano bottom-up da ripetute interazioni locali di agenti autonomi all'interno di istituzioni socio-economiche, piuttosto che da norme imposte top-down, da meccanismi artificiali di coordinamento o da assunti su agenti rappresentativi". (Gottardi e Babetto, 2006, pag. 2).

Come emerge chiaramente anche dalla precedente citazione, un tratto caratteristico di questa impostazione metodologica consiste nel fatto che, a differenza dei tradizionali modelli neoclassici che procedono a partire da regole generali e da queste derivano il comportamento delle singole unità che compongono il modello attraverso quindi una procedura top-down, i modelli ACE sono costruiti attraverso una strategia bottom-up perché il comportamento del complesso viene derivato a partire da quello dei singoli agenti. Sulla base delle regole di apprendimento stabilite è possibile, ad esempio, studiare la dinamica attraverso la quale gli agenti modificano il proprio comportamento.

La costruzione di questo tipo di modelli non è ovviamente banale e può richiedere l'utilizzo di sofisticati protocolli di programmazione e l'adozione di schemi di riferimento che chiariscano il ruolo dell'ambiente, degli agenti, delle loro regole di comportamento e le modalità di modificazione di tali regole (reti neurali, algoritmi genetici, ecc.). Essi hanno però una grande potenzialità perché consentono di tener conto della complessità del contesto in cui operano gli agenti. In sintesi, la simulazione con l'utilizzo del computer nella Agent-based Computational Economics consente di tener conto di molti aspetti della complessità della realtà economica unendo alla formalizzazione la flessibilità (rappresentazione formale flessibile). Dunque con i modelli di simulazione, elaborati attraverso il disegno del comportamento dei singoli agenti operanti in ambienti complessi, si possono ottenere reali contributi in termini di comprensione dei fenomeni economici. Non solo si riesce a riprodurre il mondo "così come è" ma, attribuendo determinati valori ai parametri, si può anche studiare il mondo "come potrebbe essere", riproducendo ad 
arte situazioni che sino ad oggi non si sono presentate ma che potrebbero presentarsi in futuro (Terna, 2003). La principale difficoltà di una metodologia bottom-up risiede nell'identificazione delle regole e, spesso, nell'elevato numero di tali regole. Il rifiuto di alcune di esse inficia ovviamente i risultati raggiunti.

\section{CONCLUSIONI: L'ECONOMIA COME VERA SCIENZA SOCIALE}

In questo saggio abbiamo esaminato il contributo della matematica alla scienza economica nel corso degli ultimi sessant'anni. La nostra riflessione non ha inteso affatto sminuire l'importanza degli studi quantitativi condotti nell'ambito della teoria economica negli ultimi decenni. E' infatti innegabile che questi studi hanno condotto ad una maggiore comprensione di molti fenomeni economici e hanno così consentito alla società di conseguire significativi guadagni di utilità nell'organizzazione della vita di tutti i giorni. In riferimento ai miglioramenti conseguiti è però necessario fare due precisazioni. La prima è che questi risultati sono stati ottenuti non solo in virtù di un sempre maggiore rigore metodologico ma anche grazie ad un confronto intellettuale sempre vivo e vivace tra varie scuole di pensiero. La conservazione di una pluralità di pensieri ed impostazioni è dunque una ricchezza ed un valore assoluto, anche dal punto di vista meramente pratico, di ogni teoria. Un valore che deve essere perciò salvaguardato contro ogni tentativo di omologazione.

La seconda riflessione che è necessario compiere per meglio comprendere i risultati conseguiti, così come anche quelli che invece non sono stati raggiunti dalla teoria economica nell'ultimo mezzo secolo, fa riferimento alle specifiche caratteristiche dell'impostazione predominante in questo periodo, vale a dire l'impostazione (o scuola) neoclassica. Questo approccio, caratterizzato dall'uso massiccio della matemati$\mathrm{ca}$, si fonda su un concetto di bomo oeconomicus la cui razionalità (o meglio iper-razionalità) si sostanzia, in pratica esclusivamente, in una straordinaria capacità di perseguire scientificamente e sistematicamente il proprio interesse personale. Si tratta dunque di un uomo simultaneamente sommamente razionale ma anche sommamente egoista. Data la manifesta profonda limitatezza di una tale rappresentazione dell'essere umano, non è difficile comprendere come molte delle insoddisfazioni riportate nella Sezione 2 del nostro saggio siano in realtà da ascri- 
vere in massima parte ad un modello di individuo che può essere considerato, al più, una caricatura mal riuscita della ricchezza e della complessità della vera umanità.

Il punto è che, a nostro avviso, se non si vuole ridurre la scienza economica ad una disciplina volta alla mera ricerca della modalità di accrescimento delle utilità individuali, è necessario definire in termini più alti e generali quale deve essere la vera finalità dell'economia e avere ben chiaro che lo sviluppo integrale dell'uomo deve essere l'obiettivo prioritario di ogni teoria economica. Si tratta, in altri termini, di perseguire il bene comune attraverso una cultura delle opere ispirate dai principi. Un autentico sviluppo è possibile solo quando alla chiarezza dei principi si associ la concretezza del fare, e se i principi devono prendere ispirazione dagli ideali, è la concretezza che riesce a condurre alle opere. In termini più specifici vogliamo qui menzionare una recente impostazione di teoria economica concepita da Alberto Quadrio Curzio, uno dei più autorevoli e conosciuti economisti italiani, che nel corso degli ultimi due decenni ha saputo costruire un compiuto paradigma metodologico, per molti versi alternativo alla teoria economica neoclassica, elaborando un originale articolato modello di sviluppo economico basato sui principi della dottrina sociale cattolica. ${ }^{5}$ Scrive, in particolare, Quadrio Curzio:

"L'economia è una scienza complessa che serve allo sviluppo economico e civile". (A. Quadrio Curzio, 2007).

E' una premessa di metodo, con anche un preciso contenuto di merito, alla quale ci sentiamo di aderire pienamente. D'altronde, che l'economia politica sia (e debba essere) una vera scienza sociale non è una novità perché questa è anche l'impostazione caratteristica di tutta una tradizione italiana, a cominciare dai grandi riformatori milanesi come Verri, Beccaria e Cattaneo.

Se dunque aderiamo alla precedente definizione di economia, dobbiamo chiederci, riprendendo le argomentazioni della precedente

5 Non possiamo ovviamente in questa sede soffermarci sulle caratteristiche specifiche di questo modello. Per una sintesi efficace di questa impostazione di teoria economica, si vedano: A. Quadrio Curzio 2001, 2002, 2005, 2007; ed anche A. Quadrio Curzio, G. Marseguerra G 2005, 2006, 2007. 
Sezione 2: può una scienza sociale ridursi al racconto di favole? può una scienza sociale non misurarsi con la realtà? $\mathrm{E}$ ancora: può una disciplina rinunciare al confronto tra impostazioni alternative? Sono tutti interrogativi ai quali ci sentiamo di rispondere negativamente, senza per questo schierarci per una teoria economica senza matematica. Semmai la nostra opinione è che, focalizzandosi sul metodo (matematico), gli economisti stanno rischiando di perdere il loro campo di indagine.

La teoria economica dell'ultimo secolo ha cercato di elaborare schemi generali nei quali far rientrare i processi economici e dai quali derivare ogni possibile conseguenza economica. I risultati sono stati notevoli ma ancora ampiamente insoddisfacenti quando si definisca nel modo corretto l'ambito e lo scopo della scienza economica. Non è dunque un caso che sia oggi molto avvertita la necessità di cambiare rotta ed elaborare nuovi paradigmi. Si pone infatti un problema antropologico: la teoria dovrebbe cercare di porre al centro dello studio l'uomo come veramente è, e cercare di capire come realmente agisce e come effettivamente si comporta. Quello che serve, in altri termini, è un modello di uomo molto più ricco e articolato dell'bomo oeconomicus neoclassico. La nostra conclusione è dunque che gli studi economici stanno procedendo verso un nuovo paradigma interpretativo della realtà economica, un paradigma in larga parte ancora da sviluppare, caratterizzato, rispetto ai canoni tradizionali neoclassici, da due tipi di innovazione: innovazione nel metodo, con una forte enfasi sui principi e con un approccio fortemente multi-disciplinare, e innovazione nell'oggetto di studio, con una marcata focalizzazione sull'essere umano e sulla sua complessità. In questa prospettiva, l'utilizzo della matematica non dovrà essere affatto abbandonato, ma dovrà invece essere potenziato e riscoperto come potente strumento di indagine fondato su un uso intelligente degli algoritmi e della potenza di calcolo dei computer. 


\section{BIBLIOGRAFIA}

Allais, M, 1989, My Life Philosophy, The American Economist, Fall, 3-17.

Blaug, M., (1997), Ugly Currents in Modern Economics, Policy Options, September, pp. 3-8.

Debreu, G., 1987, "Mathematical economics", in J. Eatwell, M. Milgate and P. Newman (eds.), The New Palgrave: A Dictionary of Economics, Macmillan, London, pp. 399-404; ediz. orig. 1986.

Fourier, J.B., 1822, Théorie analytique de la chaleur, Hermann, Parigi.

Gottardo, G e Babetto, C., 2006, Teorie, modelli evolutivi e uso della simulazione in economia. E' un approccio realmente praticabile?, paper presentato alla XXVII Riunione scientifica dell'Associazione Italiana di Ingegneria gestionale, Roma, 12-13 ottobre 2006.

Hey, J., (1997), The Economic Journal. Report of the Managing Editor, Royal Economic Society Newsletter, January.

Keynes, J. M. 1933. Essays in Biography, Rupert Hart-Davis, London, 1951.

Keynes J.M. (1936), The General Theory of Employment, Interest and Money, Macmillan, Londra.

Malinvaud, E., (1991), The Next Fifty Years, The Economic Journal, Centenary Issue, vol. 101, N. 404, January, pp. 64-68.

Manara, C.F. e Nicola, P.C., 1967, Elementi di economia matematica, Editrice Viscontea, Milano.

Morishima, M., The Good and Bad Uses of Mathematics, in Economics in Disarray, edited by P. Wiles and G. Routh. Oxford, Blackwell, 1984.

Newman, J. R., 1956, The World of Mathematics, New York.

Nicola, P.C., 1983, Economia matematica, UTET, Torino.

Nicola, P.C., 2000, Mainstream Mathematical Economics in The $20^{\text {th }}$ Century, SpringerVerlag, Berlino e New York.

Oström, T., 1988, Computer simulation: the third symbol system. Journal of Experimental Social Psychology, N. 24, pp. 381-392.

Pasinetti, L.L., 2003, Sraffa e la matematica: diffidenza e necessità - quali sviluppi per il futuro?, Accademia Nazionale dei Lincei, Convegno internazionale: Piero Sraffa, 11-12 febbraio 2003.

Quadrio Curzio, A., 2007, Economisti ed economia, il Mulino, Bologna.

Quadrio Curzio A, 2005, Ricerca e sviluppo: riflessioni su nuovi paradigmi economici per l'Unione Europea, in Rendiconti della Classe di Scienze morali, storiche e filologiche della Accademia Nazionale dei Lincei, serie IX, volume XVI, fascicolo 3, Roma, pp. 525-533.

Quadrio Curzio, A., (2002), Sussidiarietà e Sviluppo. Paradigmi per l'Europa e per l'Italia, Vita e Pensiero, Milano. 
Quadrio Curzio, A, 2001, Globalizzazione e solidarietà. Dalle Istituzioni economiche nazionali a quelle sovranazionali, in Istituto Veneto di Scienze, Lettere ed Arti, Dignità del vivere, Zadig Editore, Milano, 2001, pp.47-64.

Quadrio Curzio A. e Marseguerra G., 2007, Introduzione: Intrapresa, Sussidiarietà, Sviluppo, in Intrapresa, Sussidiarietà, Sviluppo, (a cura di A. Quadrio Curzio e G. Marseguerra), Milano, Edizioni Scheiwiller, pp. 119-132.

Quadrio Curzio A. e Marseguerra G., 2006, Introduzione: Europa, diritti umani, sviluppo equo in The World System in the 21st Century: Subsidiarity and Cooperation for Development (a cura di A. Quadrio Curzio e G. Marseguerra), Milano, Edizioni Scheiwiller, pp. 9 - 26.

Quadrio Curzio A. e Marseguerra G., 2005, Introduction: Globalization, Subsidiarity and Development, in Confronting Globalization: Global Governance and the Politics of Development (a cura di G. Marseguerra), Milano. Edizioni Scheiwiller. pp. $41-53$.

Rubinstein, A., 2006, Dilemmas of an Economic Theorist, Econometrica, 74, pp. 865 883.

Stigler, G., 1949, The Mathematical Method in Economics, in Five Lectures on Economic Problems, London, London School of Economics.

Sylos Labini, P, 2002, Il Mestiere dell'economista tra Analisi Teorica e Impegno Sociale, Quaderno n. 52, Dipartimento di Economia, Università Milano Bicocca (a cura di Giuliana Arena).

Terna, P., 2005, Economia e sistemi complessi. In R. Viale (a cura di), Le nuove economie. Milano, Il Sole 24 Ore.

Terna, P., 2003, La simulazione come strumento di indagine per l'economia. Sistemi intelligenti, 2, XV, pp. 347-376.

Terna, P., Boero, R., Morini, M. e Sonnessa, M., 2006, Modelli per la complessità - La simulazione ad agenti in economia, il Mulino, Bologna. 